\title{
Cosmological Constraints on Bose-Einstein-Condensed Scalar Field Dark Matter
}

\section{Bohua $\mathbf{L i}^{* \dagger}$}

The University of Texas at Austin

E-mail: bohualieastro.as.utexas.edu

\section{Tanja Rindler-Daller}

University of Michigan

E-mail: daller@umich.edu

\section{Paul R. Shapiro}

The University of Texas at Austin

E-mail: shapirodastro.as.utexas.edu

\begin{abstract}
We focus on the hypothesis that the dark matter is comprised of ultralight bosons that form a BoseEinstein Condensate (BEC), described by a complex scalar field. We calculate the evolution of the Friedmann-Robertson-Walker (FRW) universe in the presence of the BEC scalar field dark matter (SFDM). We find that, while WIMP CDM is non-relativistic at all times after it decouples, the equation of state of SFDM is found to be relativistic at early times, evolving from stiff $(\bar{p}=\bar{\rho})$ to radiation-like $(\bar{p}=\bar{\rho} / 3)$, before it becomes non-relativistic and CDM-like at late times $(\bar{p}=0)$. The stiff phase is a distinctive feature of our model. The timing of the transitions between these phases and regimes is shown to yield fundamental constraints on the SFDM model parameters, particle mass $m$ and self-interaction coupling strength $\lambda$. We show that SFDM is compatible with observations of the evolving background universe, by deriving the range of particle parameters required to match observations of the cosmic microwave background (CMB) and the abundances of the light elements produced by Big Bang nucleosynthesis (BBN), including $N_{\text {eff }}$, the effective number of neutrino species, and the epoch of matter-radiation equality $z_{\mathrm{eq}}$. This yields $m \geq 2.4 \times$ $10^{-21} \mathrm{eV} / \mathrm{c}^{2}$ and $9.5 \times 10^{-19} \mathrm{eV}^{-1} \mathrm{~cm}^{3} \leq \lambda /\left(\mathrm{mc}^{2}\right)^{2} \leq 4 \times 10^{-17} \mathrm{eV}^{-1} \mathrm{~cm}^{3}$. Indeed, our model can accommodate current observations in which $N_{\text {eff }}$ is higher at the BBN epoch than at $z_{\text {eq }}$, probed by the $\mathrm{CMB}$, which is otherwise unexplained by the standard CDM model involving WIMPs.
\end{abstract}

Frank N. Bash Symposium 2013: New Horizons in Astronomy

October 6-8, 2013

Austin, Texas

\footnotetext{
*Speaker.

†This work is based on the collaboration with Dr. Tanja Rindler-Daller and Dr. Paul Shapiro. A paper with the same title has been submitted to Physical Review D by the collaboration. arXiv: 1310.6061
} 
Despite the great successes of the Cold Dark Matter (CDM) model in explaining a wide range of observations of the global evolution and the formation of galaxies and large-scale structure in the Universe, the origin and microscopic nature of dark matter is still unknown. The most common form of CDM considered to-date is that of Weakly Interacting Massive Particles (WIMPs), but, so far, attempts to detect WIMPs directly or indirectly have not yet succeeded, and the allowed range of particle parameters has been significantly restricted. Some of the cosmological predictions for this kind of CDM are even in apparent conflict with observations (e.g. cuspy-cored halos and an overabundance of satellite dwarf galaxies). For these reasons, it is important to consider the consequences of different forms of CDM.

We assume that the dark matter particles are described by a spin-0 complex scalar field ('scalar field dark matter', for short; henceforth, SFDM). Several fundamental scalar fields have been predicted by a variety of unification theories, e.g., string theories and other multi-dimensional theories. The bosonic particles envisaged are typically ultralight, with masses down to the order of $10^{-33}$ $\mathrm{eV} / c^{2}$. This suggests an ultrahigh phase-space density, leading to the possibility of formation of a Bose-Einstein condensate (BEC), i.e., a macroscopic occupancy of the many-body ground state. As a result, SFDM can be described by a classic scalar field theory. The Lagrangian density we choose is

$$
\mathscr{L}=\frac{\hbar^{2}}{2 m} g^{\mu v} \partial_{\mu} \psi^{*} \partial_{v} \psi-V(\psi)
$$

where the potential above contains a quadratic term accounting for the rest-mass plus a quartic term accounting for the self-interaction

$$
V(\psi)=\frac{1}{2} m c^{2}|\psi|^{2}+\frac{\lambda}{2}|\psi|^{4} .
$$

In this work we consider a homogeneous Universe with the same cosmic inventory as the basic $\Lambda$ CDM model except that CDM is replaced by SFDM (we call it $\Lambda$ SFDM model), adopting the Friedmann-Robertson-Walker metric. We solve the Friedmann equation combined with the Klein-Gordon equation, which governs the evolution of SFDM. We pick the following fiducial values for particle mass and self-interaction coupling strength, and integrate backward in time with present-day cosmological parameters.

$$
\begin{aligned}
& (m, \lambda)_{\text {fiducial }}=\left(3 \times 10^{-21} \mathrm{eV} / \mathrm{c}^{2}, 1.8 \times 10^{-59} \mathrm{eV} \mathrm{cm}^{3}\right), \\
& \lambda /\left(m c^{2}\right)^{2}=2 \times 10^{-18} \mathrm{eV}^{-1} \mathrm{~cm}^{3} .
\end{aligned}
$$

We obtain the evolution of the homogeneous $\Lambda$ SFDM model starting from the Big Bang, as shown in the left plot of Figure 1. We indeed identify three evolutionary phases of SFDM: the initial stiff phase $\bar{\rho}_{\mathrm{SFDM}} \propto a^{-6}$, the intermediate radiation-like phase $\bar{\rho}_{\mathrm{SFDM}} \propto a^{-4}$ and the final CDM-like phase $\bar{\rho}_{\text {SFDM }} \propto a^{-3}$.

The $\Lambda$ SFDM model is subject to two constraints from observations of the homogeneous background universe. First, the transition of SFDM from the radiation-like phase to the CDM-like phase must happen early enough to be in agreement with the redshift of matter-radiation equality $z_{\mathrm{eq}}$ determined by the CMB temperature power spectrum. Second, the amount of relativistic component during BBN, which has a contribution from SFDM, either in the stiff phase or in the radiation-like phase, is constrained by the observed primordial abundances of the BBN products, parametrized by 

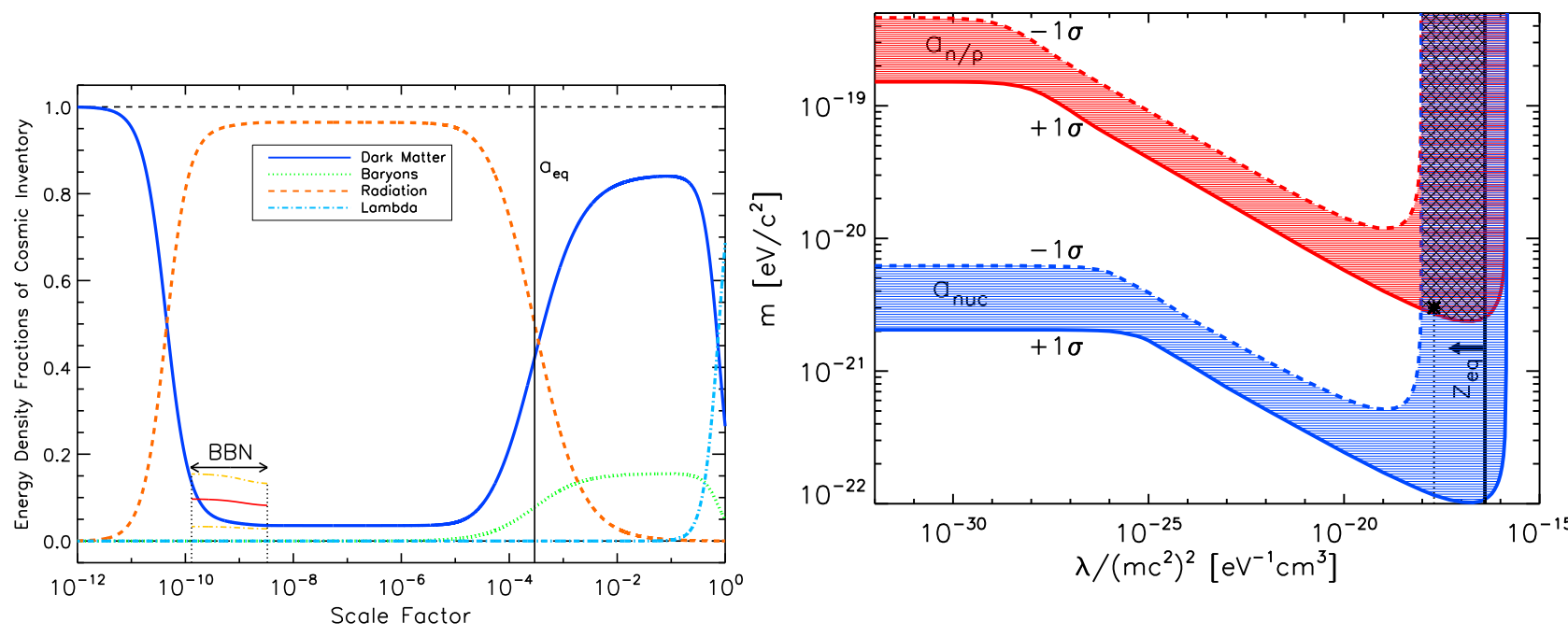

Figure 1: Left: Evolution of the fractions $\Omega_{i}$ of the energy density of each cosmic component $i$ with SFDM of mass $m=3 \times 10^{-21} \mathrm{eV} / c^{2}$ and self-interaction $\lambda /\left(m c^{2}\right)^{2}=2 \times 10^{-18} \mathrm{eV}^{-1} \mathrm{~cm}^{3}$ (fiducial model) represented by the thick curves. Different components are depicted with different line styles, as labeled in the legend. The solid vertical line corresponds to $a_{\text {eq }}$, the epoch of matter-radiation equality. On the lower left part of the figure, the thin curves represent the constraint from BBN. The solid one refers to a universe with a constant $N_{\text {eff }}$ of the central value in $N_{\text {eff }}=3.71_{-0.45}^{+0.47}$, and the two dash-dotted ones refer to such universes with $N_{\text {eff }}$ of the $1 \sigma$ limits there. The dotted vertical lines indicate the beginning of the neutron-proton ratio freezeout $a_{\mathrm{n} / \mathrm{p}}$ and the epoch of light-element production $a_{\mathrm{nuc}}$, respectively. Right: Parameter space of SFDM $\left(\lambda /\left(m c^{2}\right)^{2}, m\right)$. The solid vertical line represent the upper bound on $\lambda /\left(m c^{2}\right)^{2}$ which makes SFDM complete its transition from radiation-like to CDM-like (i.e. $\langle\bar{w}\rangle=0.001$ ) just before the observed $z_{\text {eq. }}$. The arrow indicates that the region on the left side of the solid vertical line is allowed by this constraint from $z_{\text {eq }}$. The two shaded bands are the allowed regions derived from the constraints that $N_{\text {eff }}$ be within the $1 \sigma$ interval of the value measured by BBN, at $a_{\mathrm{n} / \mathrm{p}}$ and $a_{\mathrm{nuc}}$, as labeled respectively. For each band, the thick solid (dashed) boundary curve corresponds to the upper (lower) $1 \sigma$ limit of the measured value of $N_{\text {eff }}$ mentioned above. The final allowed region is crosshatched, after combining all constraints. Our fiducial model, indicated by the star at $m=3 \times 10^{-21} \mathrm{eV} / \mathrm{c}^{2}$, lies on the dotted vertical line at $\lambda /\left(m c^{2}\right)^{2}=2 \times 10^{-18} \mathrm{eV}^{-1} \mathrm{~cm}^{3}$.

$N_{\text {eff }}$, the effective number of relativistic neutrino species. From these two constraints we construct the contour plot for the allowed SFDM parameter space, as shown in the right plot of Figure 1. We find that $m \geq 2.4 \times 10^{-21} \mathrm{eV} / c^{2}$ and $9.5 \times 10^{-19} \mathrm{eV}^{-1} \mathrm{~cm}^{3} \leq \lambda /\left(\mathrm{mc}^{2}\right)^{2} \leq 4 \times 10^{-17} \mathrm{eV}^{-1} \mathrm{~cm}^{3}$. Furthermore, we note that, due to its phase transitions, SFDM naturally provides an explanation for the difference between the $N_{\text {eff }}$ values currently measured from BBN and CMB, in which the $\mathrm{BBN}$ value is larger than the $\mathrm{CMB}$ value.

\section{References}

[1] Li, B., Rindler-Daller, T. \& Shapiro, P. R. 2013, arXiv:1310.6061 